\title{
IgG surfaces as an important component in mucosal protection
}

\begin{abstract}
New reports showing that passively administered neutralizing lgGs prevent mucosal HIV transmission in rhesus macaques or macaque neonates support the inclusion of neutralizing antibodies in vaccine design and suggest clinical strategies against mother-to-infant transmission (pages 200-210).
\end{abstract}

W ORLDWIDE, MOST HUMAN immunodeficiency virus (HIV) transmissions occur across vaginal or rectal surfaces, so an effective vaccine for AIDS will require strong mucosal immunity. Development of an efficacious mucosal vaccine is hampered by the lack of knowledge concerning mechanisms of mucosal HIV transmission and the immune responses necessary to control such infections. Two papers in this issue of Nature Medicine address the latter question. Although secretory immunoglobulin A (SIgA) generally provides the primary defense against mucosal pathogens ${ }^{1}$, Mascola et al. ${ }^{2}$ clearly show that passive administration to rhesus macaques of a mixture of anti-HIV neutralizing IgGs prevented vaginal transmission of the pathogenic simian-human immunodeficiency virus chimera SHIV-89.6PD. These results indicate that vaccines that elicit systemic, hightitered and broadly neutralizing antibodies may provide effective protection against vaginal and perhaps other routes of mucosal HIV transmission. Baba et al. ${ }^{3}$ similarly show that neutralizing IgGs, passively administered to neonatal macaques before and after infection protect against the orally administered chimera SHIV-vpu+, suggesting a potentially effective strategy for inhibiting vertical HIV transmission

from mothers to infants. Neither paper, however, reports the mechanism(s) by which the IgG antibodies mediate mucosal protection.

Passive infusion of rhesus macaques with a mixture of IgG1 monoclonal antibodies including 2F5 and 2G12, both capable of neutralizing a broad spectrum of primary HIV isolates ${ }^{4}$, and HIVIG, a purified polyclonal IgG preparation obtained from the plasma of HIV-1-positive individuals, prevented intravenous SHIV-89.6PD

\section{MARJORIE ROBERT-GUROFF}

infection of some animals ${ }^{5}$. Extending these findings, passive infusion of one, two or three of these antibodies 24 hours before vaginal challenge with SHIV89.6PD has now conferred complete protection in eight of fourteen macaques ${ }^{2}$. The remaining six macaques showed diminished viremia and unchanged or only moderate reductions in CD4 counts compared with those of naive controls. The results indicate that it may be easier to protect against vaginal than intravenous

days before birth and to neonates 1 hour before and 8 days after virus challenge, protected four of four newborns from oral infection by SHIV-vpu+. This extends previous studies showing passive protection of neonates by hyperimmune rhesus macaque serum ${ }^{6}$. However, the inability of the hyperimmune serum to neutralize the $\operatorname{SIV}_{251}$ challenge virus and its high RANTES content made it impossible to determine whether antibody alone mediated the protection.

Although both studies show that IgG antibodies can provide mucosal protection, both have caveats. To ensure infection of all controls with SHIV-89.6PD, Mascola et al. treated the macaques with progesterone, which causes thinning of the vaginal epithelium, and may have also allowed greater transudation of IgG into the vaginal fluid. In the absence of hormone treatment, the extent of protection might not be so great. Therefore, one must temper the suggestion indicated by their results that systemic inहु $\bar{\xi}$ duction of high-titered antibodies would uniformly result in vaginal protection or even protection at other mucosal surfaces.

Baba et al. prevented oral virus transmission to neonates by maintaining protective IgG levels 3 weeks after birth by additional antibody adminis-

infection; however, the small number of animals involved make the suggestion only speculative.

Baba et al. report protection of rhesus macaque mothers against intravenous challenge with SHIV-vpu+ after passive administration of the F105 IgG1 monoclonal antibody together with 2G12 and 2F5. Although this observation itself is not new, the work is new in that it shows that three doses of the IgG1 neutralizing antibodies alone, administered to mothers 5 trations. Although protective IgG levels were found in cord blood, it remains to be determined whether a single passive immunization of the mothers would have sufficed for oral protection of the newborns. In addition, it may have been relatively easy to protect against SHIV-vpu+. The extent to which protection against more pathogenic viruses would occur is not known. SHIV-vpu+ is readily neutralized, replicates poorly in macaques, and has elicited disease only in a single 
neonate and after prolonged infection ${ }^{7}$

Finally, it is not clear if sterilizing protection was achieved, or if viral replication was simply suppressed to undetectable levels, as crucial viral RNA determinations have not yet been made. It is possible that cells were initially infected in the oral mucosa and traveled to secondary lymphoid sites, where the continuous high level of neutralizing antibody and other immune effector mechanisms led to apparent virus clearance. Nevertheless, the importance of this study lies in the demonstration

\section{Ways that IgGs might prevent mucosal transmission}

- Prevention of binding or attachment.

- Trapping in mucus.

- Viral neutralization.

- Inhibition of dendritic cell or M cell infection.

- Complement activation of antibodydependent cell-mediated cytotoxicity leading to lysis of virus or infected cells .

- Inhibition of transport or transcytosis to T cells.

- Neutralization at a distant site.

that passive administration of potent neutralizing IgGs can prevent HIV transmission from mothers to their offspring, suggesting a potential clinical utility.

In general, the ability of viral-specific IgG to protect against infection at the mucosal surface is relatively limited. It can neutralize virus, mediate antibody-dependent cellular cytotoxicity and activate complement. The last two activities were not explored in the studies reported here. Unlike SIgA, IgG is poorly equipped to repel viral attachment to the mucosal surface, or to trap viral particles in mucus.

Thus, the mechanism(s) of the observed protection remains obscure. Target cells for vaginal and oral HIV and SIV infections probably differ, as do the epithelial coverings at these sites. In the vagina and tonsils, stratified epithelial barriers contain dendritic cells that can carry virus to $\mathrm{T}$ cells at local and distant sites. Deep crypts in the palatine and sublingual tonsils contain $\mathrm{M}$ cells that can transport viral particles to underlying $\mathrm{T}$ cells and may be susceptible to HIV infection ${ }^{8}$. Whether neutralizing antibody can block M-cell infection is unknown, but it can block HIV infection of dendritic cells, and also viral transmission to T cells ${ }^{9}$. However, the levels of $2 \mathrm{G} 12$ and $2 \mathrm{~F} 5 \mathrm{IgG}$ in vaginal fluids were probably insufficient to neutralize the challenge virus, and the serum neutralizing antibody levels present at challenge did not correlate with the protection observed ${ }^{2}$. Similarly, neutralizing antibody activity was not detected in the saliva of passively immunized neonates ${ }^{3}$. Thus, it seems unlikely that the mechanism of protection was neutralization of initial cell infection. After application of SIV to tonsilar epithelium, T cells in the organized mucosa-associated lymphoid tissue rather than dendritic cells were shown to be first infected, with rapid spread to other lymphoid tissues ${ }^{10}$. Perhaps neutralization at secondary lymphoid sites was important here in controlling vaginal and oral transmission.

Regardless of the mechanism, these reports demonstrate that HIV-specific IgG can be much more important in mucosal protection than previously thought. Elicitation of potent neutralizing IgG antibodies should remain an important component of broadly protective vaccines.

1. Russell, M.W., Kilian, M., \& Lamm, M.E. in Mucosal Immunology (eds. Ogra, P.L. et al.) 225-240 (Academic Press, San Diego, 1999).

2. Mascola, J.R. et al. Protection of macaques against vaginal transmission of a pathogenic HIV-1/SIV

\section{Gut reaction}

The enteric nervous system of the gut contains as many neurons as the spinal cord, and many chemical messengers that regulate feeding by the brain have similar functions in the gut. In the December 1999 issue of Neuron, Annette Kirchgessner and Min-tsai Liu (State University of New York, Brooklyn) reported that some enteric neurons contain orexin, a peptide also found in the hypothalamus, where it stimulates feeding behavior and regulates energy homeostasis. In rat, human, guinea pig and mouse, neurons expressing orexin protein were found throughout the small intestine, in the stomach, and in the colon. All of these orexin-containing neurons also had receptors for leptin, a circulating peptide that regulates food intake, indicating that they may be responsive to extracellular glucose concentration. These neurons responded to fasting by increasing levels of orexin protein. As shown in the photo, 2 days of food deprivation also upregulated the phosphorylated (activated) form of cyclic AMP response element-binding protein (red) in the nucleus of orexin-containing (green) submucosal neurons from guinea pig ileum. Application of orexin excited secretomotor neurons in the guinea pig submucosal plexus and increased intestinal motility. The authors concluded that orexin may be an important regulator of energy homeostasis in the gut. These findings also support the more speculative idea that enteric neuron microcircuits may be involved in regulating feeding behavior. In future experiments, the authors plan to investigate whether these orexin-containing neurons sense the metabolic state of the animal. Orexins may be the peripheral factor that signals the brain to initiate feeding in response to glucose deprivation. If so, defects in this system might contribute to obesity, making orexin receptors a potential target for obesity-reducing drugs.

Sandra Aamodt 